\title{
ENSAIOS CLIINICOS RANDOMIZADOS NA ORTOPEDIA E TRAUMATOLOGIA: AVALIAÇÃO SISTEMÁTICA DA EVIDÊNCIA NACIONAL
}

\author{
RANDOMIZED CONTROLLED TRIALS IN ORTHOPEDICS \\ AND TRAUMATOLOGY: SYSTEMATIC ANALYSIS OF THE NATIONAL EVIDENCE
}

Vinícius Ynoe de Moraes ${ }^{1}$, Cesar Domingues Moreira' ${ }^{1}$ Marcel Jun Sugawara Tamaoki², Flávio Faloppa ${ }^{4}$, João Carlos Belloti ${ }^{3}$

\section{RESUMO}

Objetivo: Verificar se houve melhora da qualidade e quantidade dos ensaios clínicos randomizados (ECRs) publicados na literatura nacional, por meio da aplicação de escores estruturados e validados na literatura. Métodos: Selecionamos, eletronicamente, todas as edições das duas revistas indexadas e de escopo ortopédico da literatura nacional - Acta Ortopédica Brasileira (AOB) e Revista Brasileira de Ortopedia (RBO), no período de 2000-2009, todos os ECRs publicados. Dois pesquisadores independentes identificaram e pontuaram os ECRs de acordo com as escalas de JADAD e do grupo de trauma musculoesquelético da Colaboração Cochrane. Os trabalhos selecionados foram agrupados: 1) pelo período de publicação 2000-2004 e 2004-2009; 2) periódico de publicação (AOB e RBO). Resultados: Vinte e dois trabalhos foram selecionados, sendo10 da AOB e 12 da RBO. Não houve diferença entre o número proporcional (nECR/nTotal de trabalhos publicados) de ensaios clínicos randomizados publicados nos dois periódicos ( $\mathrm{p}=0,458)$, assim como para os escores de JADAD ( $\mathrm{p}=0,722)$ e da Colaboração Cochrane $(\mathrm{p}=$ 0,630). Conclusão: A qualidade e quantidade relativa de ensaios clínicos randomizados nos periódicos analisados foi semelhante. Há uma tendência de melhora da qualidade; contudo, não há acréscimo da quantidade de ensaios clínicos randomizados nos dois períodos analisados.

Descritores - Medicina Baseada em Evidências; Ensaios Clínicos Randomizados; Metodologia; Ortopedia; Traumatologia

\section{ABSTRACT}

Objective: To assess whether there was an improvement in the quality and quantity of randomized controlled trials (RCTs) in nationally published journals through an application of standardized and validated scores. Methods: We selected, electronically, for the period of 2000-2009, all RCTs published at the two indexed, orthopaedics-focused Brazilian journals: Acta Ortopédica Brasileira(AOB) and Revista Brasileira de Ortopedia (RBO). These RCTs were identified and scored by two independent researchers according to the JADAD scale and Cochrane Bone, Joint and Muscle Trauma Group score. The selected studies were grouped: 1) by publication period (2000-2004 and 2004-2009); 2) journal of publication (AOB and RBO). Results: Twenty-two papers were selected, 10 from $A O B$ and 12 from RBO. No statistically significant differences were found between the proportion (nRCT/nTotal of published papers) of RCTs published in the two journals $(p=0.458)$, as well as for the JADAD score $(p=0.722)$ and Cochrane score $(p=0.630)$. Conclusion: The quality and quantity of randomized clinical trials in the period was similar in the journals analyzed. There is a trend of improvement of quality, yet there was no increase in the number of randomized clinical trials in both periods.

Keywords - Evidence-Based Medicine; Randomized Controlled Trials; Methodology; Orthopedics; Traumatology

\footnotetext{
1 - Residente do terceiro ano do Departamento de Ortopedia e Traumatologia - Universidade Federal de São Paulo, Unifesp.

2 - Médico do Setor de Ombro e Cotovelo da Disciplina de Mão e Membro Superior do Departamento de Ortopedia e Traumatologia

- Universidade Federal de São Paulo, Unifesp.

3 - Professor Adjunto do Departamento de Ortopedia e Traumatologia - Universidade Federal de São Paulo, Unifesp.

4 - Professor Titular do Departamento de Ortopedia e Traumatologia - Universidade Federal de São Paulo, Unifesp.
}

Trabalho realizado na Escola Paulista de Medicina, Universidade Federal de São Paulo - Unifesp.

Correspondência: Rua Borges Lagoa, 593, 5o. andar. São Paulo-SP - E-mail: vym70@yahoo.com.br

Trabalho recebido para publicação: 02/03/10, aceito para publicação: 09/03/10. 


\section{INTRODUÇÃO}

A avaliação crítica da literatura ortopédica tem se tornado uma ferramenta necessária para aqueles que buscam o conhecimento atualizado ${ }^{(1-3)}$. Neste contexto, a aquisição de informações com uma sistemática baseada em evidências e a consequente categorização em níveis de evidência ${ }^{(4)}$ torna-se obrigatória, haja vista o grande número de publicações e frequentes armadilhas metodológicas, que podem conduzir o leitor a conclusões falhas e potencialmente perigosas para prática médica $^{(5-8)}$.

Os esforços para se definir critérios metodológicos adequados e uniformes para publicações de estudos de boa qualidade têm levado pesquisadores a repensar o planejamento e a publicação de pesquisas para que se atinja melhor rigor científico e maior validade $\operatorname{externa}^{(9-11)}$. Dentre estas, no que diz respeito ao tratamento o ensaio clínico randomizado, é o estudo que apresenta melhor nível de evidência ${ }^{(12,13)}$, visto que seu desenho metodológico permite concluir de forma comparativa e aleatória a melhor opção de tratamento para cada questão clínica, utilizando-se ferramentas validadas para aferição dos desfechos significativos da condição clínica estudada, sendo atualmente escopo de maior prestígio e atenção da literatura.

A despeito da atribuição de níveis de evidência, não se pode deixar de avaliar os estudos de forma individualizada, haja vista a frequente inconsistência destas atribuições, explicitadas por alguns autores ${ }^{(5,14)}$. Estudo pregresso demonstrou equivalência entre a qualidade da produção internacional e nacional, no período de 1988 e $2002^{(15)}$. Neste ínterim, a literatura americana, recentemente, demonstrou uma melhora na qualidade das publicações, a despeito de um número considerável de falhas metodológicas potencialmente corrigíveis ${ }^{(9,16)}$.

Espera-se que, na literatura nacional, haja uma melhora quantitativa e qualitativa do nível de evidência dos trabalhos publicados, seguindo a tendência vista mundialmente $\mathrm{e}^{(17)}$.

\section{Os objetivos deste estudo são:}

1. Verificar se há um aumento da publicação de estudos clínicos randomizados, comparando-se os períodos de 2000-2004 e 2004-2009 - avaliação quantitativa;

2. Avaliar esses estudos de acordo com a qualidade metodológica (escore de JADAD e escore da Colaboração Cochrane) - avaliação qualitativa.

\section{MATERIAIS E MÉTODO}

A pesquisa foi feita por dois pesquisadores independentes (V.Y.M, C.D.M), que selecionaram mediante busca eletrônica todas edições e realizaram a extração manual dos estudos descritos como ensaios clínicos randomizados nas duas revistas nacionais indexadas, que apresentam como escopo principal a publicação de pesquisas relacionadas à ortopedia e traumatologia: Acta Ortopédica Brasileira (AOB) e Revista Brasileira de Ortopedia (RBO).

Definimos como ensaio clínico randomizado: I. Aquele que apresente desenho planejado antes da coleta de dados; II. Envolva tratamento; III. Apresente a possibilidade de inclusão de um paciente, com a mesma chance, em quaisquer dos grupos de alocação $^{(13)}$.

Os dois pesquisadores, de forma independente, avaliaram todos os títulos e resumos estruturados destes periódicos, separando para avaliação pormenorizada aqueles que se apresentaram como ensaios clínicos randomizados, a priori, publicados entre 2000 e 2009. Os estudos que apresentaram resumos imprecisos ou pouco conclusivos foram separados e dúvidas adjacentes foram resolvidas pela leitura do manuscrito na íntegra e/ou contato com o pesquisador principal. Estes foram incluídos ou excluídos da pesquisa assim que se obtiveram as informações pendentes e/ou após consenso dos pesquisadores.

Os estudos incluídos foram pontuados de acordo com o escore de Jadad et al $^{(18)}$ e escore do grupo de trauma musculoesquelético da colaboração Cochrane (Cochrane Bone, Joint and Muscle Trauma Group) ${ }^{(19)}$. Após a leitura na íntegra dos trabalhos, considerou-se os escores isoladamente (E1 e E2), assim como a média entre estes (E1/E2), para análise dos grupos, definidos como: Grupo I: trabalhos publicados antes de 2004; Grupo II: trabalhos publicados entre 2004-2009. Associadamente, realizou-se a avaliação de acordo com a revista de publicação (AOB e RBO), para os mesmos escores acima definidos.

Este trabalho foi aprovado pelo comitê de ética em pesquisa desta instituição $\left(n^{\circ} 0016 / 10\right)$.

\section{Critérios de inclusão}

Ensaios clínicos randomizados, publicados entre 2000 e 2009, em revistas nacionais indexadas do escopo da ortopedia e traumatologia (Acta Ortopédica Brasileira e Revista Brasileira de Ortopedia). 


\section{Critérios de exclusão}

Estudos que resultaram em dúvidas metodológicas, mesmo após reunião de consenso entre os pesquisadores, e que não se conseguiu contato com o pesquisador principal por meios tradicionais.

\section{Análise estatística}

A avaliação da premissa da normalidade da distribuição da amostra foi obtida pelo teste de SmirnovKolmogorov. Utilizamos o teste de Mann-Whitney para comparação entre as médias dos escores, nos diferentes grupos. Para avaliação da confiabilidade, com o objetivo de avaliar a consistência interna entre a avaliação dos escores de JADAD interobservadores, utilizou-se do teste Kappa de Cohen, pareados dois a dois ${ }^{(\mathbf{2 0})}$. A proposta difundida por Landis e Koch ${ }^{(21)}$ para interpretação do grau de concordância foi utilizada: I. < 0 - pobre; II. 0 a 0,20 - discreta; III. 0,21 a 0,40 - razoável; IV. 0,41 a 0,60 — moderada; V. 0,61 a 0,80 — ótima, substancial; VI. 0,81 a 1,00 — quase perfeita. O teste do Qui-quadrado foi utilizado para a avaliação proporcional entre os diferentes períodos (proporção dos ensaios clínicos randomizados no período 1 e proporção de ensaios clínicos randomizados no período 2).

\section{RESULTADOS}

Foram incluídos 22 trabalhos, sendo destes 10 provenientes da $\mathrm{AOB}^{(22-31)}$ e 12 da $\mathrm{RBO}^{(32-43)}$ (Tabela1). A avaliação da confiabilidade interobservadores (E1 e E2), para o escore de JADAD resultou em um valor de Kappa de 0,611 (substancial, ótima).

Tabela 1 - Número e porcentagem de ECRs e NECRs nos periódicos.

\begin{tabular}{c|c|c|c}
\hline & AOB & RBO & Valor de $\mathbf{P}$ \\
\hline ECR & $10(2,4 \%)$ & $12(1,7 \%)$ & \\
\hline NECR & 411 & 679 & 0,458 \\
\hline
\end{tabular}

ECR - ensaio clínico randomizado.

NECR - não ensaio clínico randomizado.

A comparação da qualidade metodológica dos trabalhos, de acordo com os escores de JADAD e da Colaboração Cochrane, não demonstrou diferenças estatísticas quando agrupados por periódico de publicação (Tabelas 2 e 3). Quando agrupados por período de publicação, não houve diferenças estatisticamente significantes (Tabelas 4 e 5), exceto para o examinador 2, para o escore de JADAD, favorecendo melhor qualidade
Tabela 2 - Médias de escore de Cochrane para os ECRs de acordo com os periódicos.

\begin{tabular}{c|c|c|c|c|c}
\hline \multirow{2}{*}{} & \multicolumn{4}{|c}{ Periódico } \\
\cline { 2 - 6 } & \multicolumn{2}{|c|}{ AOB } & \multicolumn{2}{c}{ RBO } & \\
\cline { 2 - 6 } & Média & DP & Média & DP & Valor de P \\
\hline Escore Cochrane & 12,75 & 4,63 & 14,33 & 3,93 & 0,722 \\
\hline
\end{tabular}

Tabela 3 - Médias de escore de JADAD para os ECRs de acordo com os periódicos.

\begin{tabular}{l|c|c|c|c|c|c|c}
\hline \multicolumn{2}{c|}{} & \multicolumn{2}{|c|}{ E1JADAD } & \multicolumn{2}{c|}{ E2JADAD } & \multicolumn{2}{c}{ Média (E1/E2) } \\
\cline { 3 - 8 } \multicolumn{2}{c|}{} & Média & DP & Média & DP & Média & DP \\
\hline Periódico & AOB & 2,1 & 1,29 & 2,1 & 1,45 & 2,1 & 1,31 \\
\hline & RBO & 2,5 & 1,38 & 2,17 & 1,19 & 2,33 & 1,23 \\
\hline Valor de P & & & 0,497 & & 0,923 & & 0,674 \\
\hline
\end{tabular}

Tabela 4 - Média de escore de JADAD para os ECRs nos dois períodos.

\begin{tabular}{c|c|c|c|c|c|c|c}
\hline \multicolumn{2}{c|}{} & \multicolumn{2}{c|}{ E1JADAD } & \multicolumn{2}{c|}{ E2JADAD } & \multicolumn{2}{c}{ Média (E1/E2) } \\
\cline { 3 - 8 } \multicolumn{1}{c}{} & Média & DP & Média & DP & Média & DP \\
\hline \multirow{3}{*}{ Período } & $\begin{array}{c}2000-2004 \\
(\mathrm{n}=7)\end{array}$ & 1,86 & 0,69 & 1,29 & 0,49 & 1,57 & 0,53 \\
\cline { 2 - 8 } & $\begin{array}{c}2004-2009 \\
(\mathrm{n}=15)\end{array}$ & 2,53 & 1,51 & 2,53 & 1,36 & 2,53 & 1,37 \\
\hline Valor de P & & & 0,447 & & $0,021^{*}$ & & 0,09 \\
\hline
\end{tabular}

Tabela 5 - Média de escore de Cochrane para os ECRs nos dois períodos.

\begin{tabular}{c|c|c|c|c|c}
\hline \multirow{2}{*}{} & \multicolumn{4}{|c|}{ Período } & \multirow{2}{*}{} \\
\cline { 2 - 5 } & \multicolumn{2}{|c|}{$2000-2004$} & \multicolumn{2}{|c|}{$2004-2009$} & \\
\cline { 2 - 5 } & Média & DP & Média & DP & Valor de P \\
\hline Escore Cochrane & 14,07 & 3,62 & 13,4 & 4,6 & 0,63 \\
\hline
\end{tabular}

metodológica para o período de 2004 a 2009 (teste de Mann-Whitney; $p=0,02$ ). A razão entre trabalhos publicados e ensaios clínicos randomizados não se demonstrou diferente entre os dois periódicos ( $\mathrm{p}=0,867)$.

\section{DISCUSSÃO}

Os resultados deste estudo demonstram uma tendência de melhora da qualidade metodológica dos ensaios clínicos randomizados publicados em nosso meio, a despeito de não se encontrar significância estatística para ambos os pesquisadores. Esta constatação vem de encontro aos esforços advindos de iniciativas internacionais e nacionais ${ }^{(1,2,4,10,44,45)}$. Outro fator que chama atenção é a equivalência, pelo menos ao que diz respeito 
a esta análise pontual, da qualidade metodológica de ambas as revistas nacionais.

O número absoluto de ensaios clínicos randomizados, próximos a $2 \%$, demonstram duas difíceis realidades: a primeira relaciona-se à dificuldade de se realizar ensaios randomizados e mascarados em especialidade de apelo cirúrgico e a característica de se valorizar as séries de casos, refletindo um rol de pacientes tratados de forma uniforme e sem critério metodológico, o que é de certa forma congruente com a literatura internacional, que apresenta porcentagens semelhantes de ensaios clínicos randomizados, com já demonstrada melhora do rigor metodológico ${ }^{(46,47)}$. A despeito do mérito destas, não se pode guiar terapêuticas, ao menos para doenças de sabida elevada frequência, por estudos de nível III e IV. É dentro deste panorama que podem ser criados protocolos com qualidade metodológica adequada que envolvam diversos centros de pesquisa, sugerindo-se, neste escopo, a atividade importante das sociedades de ortopedia e traumatologia $^{(48)}$.

\section{REFERÊNCIAS}

1. Reis FB, Lopes AD, Faloppa F, Ciconelli RM. A importância da qualidade dos estudos para a busca da melhor evidência. Rev Bras Ortop. 2008; 43(6):209-16.

2. Schunemann HJ, Bone L. Evidence-based orthopaedics: a primer. Clin Orthop Relat Res. 2003;(413):117-32.

3. Belangero WD. Reflexões sobre a metodologia na pesquisa em ortopedia e traumatologia. Acta Ortop Bras. 2001;9(3):59-61.

4. Camanho GL. Nível de evidência [Editorial]. Rev Bras Ortop. 2009;44(6):1-2.

5. Poolman R, Struijs P, Krips R, Sierevelt I, Lutz K, Bhandari M. Does a "Level I Evidence" rating imply high quality of reporting in orthopaedic randomised controlled trials? BMC Med Res Methodol. 2006;6:44.

6. Poolman RW, Kerkhoffs GM, Struijs PA, Bhandari M, International EvidenceBased Orthopedic Surgery Working Group. Don't be misled by the orthopedic literature: tips for critical appraisal. Acta Orthop. 2007;78(2):162-71.

7. Devereaux PJ, Choi PT, El-Dika S, Bhandari M, Montori VM, Schünemann HJ, et al. An observational study found that authors of randomized controlled trials frequently use concealment of randomization and blinding, despite the failure to report these methods. J Clin Epidemiol. 2004;57(12):1232-6.

8. Schulz KF, Chalmers I, Hayes RJ, Altman DG. Empirical evidence of bias. Dimensions of methodological quality associated with estimates of treatment effects in controlled trials. JAMA. 1995;273(5):408-12.

9. Bhandari M, Guyatt G, Lochner H, Sprague S, Tornetta P. Application of the Consolidated Standards of Reporting Trials (CONSORT) in the Fracture Care Literature. J Bone Joint Surg Am. 2002;84(3):485-9.

10. Moher D, Jones A, Lepage L, CONSORT Group (Consolitdated Standards for Reporting of Trials). Use of the CONSORT statement and quality of reports of randomized trials: a comparative before-and-after evaluation. JAMA. 2001;285(15):1992-5.

11. Vandenbroucke JP, von Elm E, Altman DG, Gøtzsche PC, Mulrow CD, Pocock SJ, et al. Strengthening the Reporting of Observational Studies in Epidemiology (STROBE): explanation and elaboration. Ann Intern Med 2007;147(8):W163-94.

12. Cochrane Handbook for Systematic Reviews of Interventions 4.2.4 [updated March 2005]. In: Higgins JPT, Green S, eds. The Cochrane Library. Chichester, UK: John Wiley \& Sons, Ltd.; 2005.
Alguns vieses podem ser considerados nesta pesquisa: a dificuldade de se utilizar as escalas de avaliação de ensaios clínicos randomizados (JADAD e Cochrane) e, devido à baixa frequência de ensaios encontrados, a possibilidade de termos cometido um erro tipo Beta. O primeiro deve-se às dificuldades inerentes aos sistemas de classificação e o segundo a aspectos de nossa realidade.

\section{CONCLUSÃO}

A qualidade e quantidade relativa de ensaios clínicos randomizados nos periódicos analisados foi semelhante.

Há uma tendência de melhora da qualidade; contudo, não há acréscimo da quantidade de ensaios clínicos randomizados nos dois períodos analisados.

Pesquisas futuras com questões clínicas de tratamento devem se focar em estimular a elaboração de protocolos de pesquisa com refinamento metodológico (ECR) com intuito de fornecer o melhor nível de evidência e instituir a cooperação interinstitucional.

13. Moher D, Pham B, Jones A, Cook DJ, Jadad AR, Moher M, et al. Does quality of reports of randomised trials affect estimates of intervention efficacy reported in meta-analyses? Lancet. 2006;352(9128):609-13.

14. Dulai SK, Slobogean BLT, Beauchamp RD, Mulpuri K. A quality assessment of randomized clinical trials in pediatric orthopaedics. J Pediatr Orthop. 2007;27(5):573-81.

15. Amatuzzi ML. Análise da evolução qualitativa de publicações em Ortopedia e Traumatologia comparação entre a "Revista Brasileira de Ortopedia" e "The Journal of Bone and Joint Surgery". Rev Bras Ortop;39(9):527-35.

16. Dijkman BG, Abouali JA, Kooistra BW, Conter HJ, Poolman RW, Kulkarni AV, et al. Twenty years of meta-analyses in orthopaedic surgery: has quality kept up with quantity? J Bone Joint Surg Am. 2010;92(1):48-57.

17. Bhandari M, Richards R, Sprague S, Schemitsch E. The quality of reporting of randomized trials in the Journal of Bone and Joint Surgery from 1988 through 2000. J Bone Joint Surg Am. 2002;84(3);388-96.

18. Jadad AR, Moore RA, Carroll D, Jenkinson C, Reynolds DJ, Gavaghan DJ, et al. Assessing the quality of reports of randomized clinical trials: is blinding necessary? Control Clin Trials. 1996;17(1):1-12.

19. Bhandari M, Morrow F, Kulkarni A, Tornetta P. Cochrane Bone, Joint and Muscle Trauma Group Meta-analyses in orthopaedic surgery. A systematic review of their methodologies. J Bone Joint Surg Am. 2006;83(1):15-24.

20. Petrie A. Statistics in orthopaedic papers. J Bone Joint Surg $\mathrm{Br}$ 2006;88(9):1.121-36.

21. Landis J, Koch G. The measurement of observer agreement for categorical data. Biometrics 1977;33:159-74.

22. Benegas E, Amódio DT, Correia LFM, Malavolta E, Ramadan LB, Ferreira Neto AA, et al. Estudo comparativo prospectivo e randomizado entre o tratamento cirúrgico das fraturas diafisárias do úmero com placa em ponte e haste intramedular bloqueada (análise preliminar). Acta Ortop Bras. 2007;15(2):87-92.

23. Cabral CMN, Melim AM, Sacco CN, Marques AP. Fisioterapia em pacientes com sìndrome fímoro-patelar: comparação de exercl̀cios em cadeia cinética aberta e fechada. Acta Ortop Bras. 2008;16(3):180-5.

24. Ferracini AM, Faloppa F, Daltro GC, Crisóstomo Júnior DC, Reis FB, Belotti JC. Estudo prospectivo e randomizado de pacientes com fraturas expostas da 
diáfise do fêmur submetidos a osteossíntese com placa e haste intramedular bloqueada a foco aberto. Acta Ortop Bras. 2008;16(2):102-6.

25. Garcia Filho RJ, Korukian M, Santos FPE, Viola DCM, Puertas EB. Ensaio clínico randomizado, duplo-cego, comparativo entre a associação de cafeína, carisoprodol, diclofenaco sódico e paracetamol e a ciclobenzaprina, para avaliação da eficácia e segurança no tratamento de pacientes com lombalgia e lombociatalgia agudas. Acta Ortop Bras. 2006;14(1):11-6.

26. Hamra A, Miguel OF, Marteli TK, Barci LP, Leme FM. DHS e OPS: estudo comparativo da falência de osteossìntese. Acta Ortop Bras. 2009; 17(2):35-9.

27. Nascimento TF, D'Elia LFB, Gonçalves LO, Dobashi ET. Estudo randomizado do tratamento cirurgico da sindrome do tunel do carpo. Acta Ortop Bras. 2007;15(4):218-21.

28. Pereira EA, Mattar Júnior R, Azze RJ. Comparative study between endoscopic technique by a proximal port and mini palmary incision in the surgical treatment of carpal tunnel syndrome. Acta Ortop Bras. 2003;11(Suppl 1):48-57.

29. Rejaili WA, Chueire AG, Cordeiro JA, Petean FC, Carvalho Filho GD. Avaliação do uso do Hylano GF-20 no pós-operatório de artroscopia de joelho por artrose. Acta Ortop Bras. 2005;13(1):20-3.

30. Silva AL, Imoto DM, Croci AT. Estudo comparativo entre a aplicação de crioterapia, cinesioterapia e ondas curtas no tratamento da osteoartrite de joelho. Acta Ortop Bras. 2007;15(4):204-9.

31. Silva NA, Marczyk LR. Eficácia e tolerabilidade da nimesulida versus celecoxib na osteoartrite. Acta Ortop Bras. 2001;9(1):42-54.

32. Carvalho Júnior LH, Denaro MF, Gonçalves TJ, Brito HMC, Gonçalves MJ. Uso do dreno de sucção após a reconstrução do ligamento cruzado anterior. Rev Bras Ortop. 41(6):211-16

33. Carvalho Júnior LH, Rezende PM, Castro CA, Soares LF, Gonçalves MJ. Bupivacaína, epinefrina e morfina na analgesia pós-artroplastia total do joelho. Rev Bras Ortop. 2006;41(9):347-51.

34. Domit Filho M, Costa do Monte AP, Nagai M, Ribeiro MV, Maciel LG, Ribas Filho $\mathrm{JM}$, et al. Estudo de posicionamento do enxerto na substituição do ligamento cruzado anterior. Rev Bras Ortop. 2002;37(4):141-50.

35. Fernandes HJ, Reis FB, Tucci Neto PF, Belangero WD. Placa em ponte e haste intramedular bloqueada: estudo comparativo no tratamento de fraturas multifragmentárias da diáfise do fêmur. Rev Bras Ortop. 2002;37(9):392-8.

36. Figueiredo EA, Neves EJ, Yoshizawa Júnior H, Dall'Ara Neto A, Nascimento
LF, Matta Faria $\mathrm{GH}$, et al. Estudo prospectivo randomizado comparativo entre os tratamentos cirúrgico utilizando placa anterior e o não cirúrgico das fraturas do terço médio da clavícula. Rev Bras Ortop. 2008;43(10):419-25.

37. Fonseca F. Mosaicoplastia revestida com periósseo no tratamento de perda osteocondral do joelho. Rev Bras Ortop. 2009;44(2):153-8.

38. Kanan PS, Schwartsmann CR, Boschin LC, Conrad S, Silva MF. Estudo comparativo entre rivaroxaban e enoxaparina na profilaxia de tromboembolismo venoso profundo em pacientes submetidos à artroplastia total do quadril. Rev Bras Ortop. 2008;43(8):319-28.

39. Almeida Filho IA, Leitão ICdS, Castro L, Pires Neto PJ. Luxação glenoumeral anterior aguda: estudo comparativo entre métodos de redução incruenta. Rev Bras Ortop. 2006;41(11/12):455-60.

40. Boschin LC, Schuck GdF, Oliveira GK, Schmirdt I, Schwartsmann CR. Artrotomia "versus" artroscopia: avaliação pós-operatória da reconstrução do ligamento cruzado anterior. Rev Bras Ortop. 2002;37(1/2):23-30.

41. Ruaro AF, Carvalho Júnior AE, Fernandes TD, Salomão O, Aguilar JA, Meyer AT. Estudo comparativo entre duas técnicas de osteotomia no tratamento do hálux valgo: análise clínica e radiográfica. Rev Bras Ortop. 2000;35(7):248-59.

42. Sawaia RN, Belangero WD. Estudo comparativo entre a técnica de miniincisão e a via de acesso a foco aberto para o tratamento das fraturas transtrocanterianas. Rev Bras Ortop. 2005;40(3):106-18.

43. Silva JB, Calcagnotto G, Oliveira CG, Fisher H. Estudo prospectivo randomizado da mobilização ativa precoce após reparo dos tendões flexores em zona 2. Rev Bras Ortop. 2003;38(10):581-8.

44. Atkins D, Eccles M, Flottorp S, Guyatt GH, Henry D, Hill S, et al. Systems for grading the quality of evidence and the strength of recommendations I: critical appraisal of existing approaches The GRADE Working Group. BMC Health Serv Res. 2004;4(1):38.

45. Wright J, Swiontkowski M, Heckman J. Introducing levels of evidence to the journal. J Bone Joint Surg Am. 2003;85(1):1-3.

46. Kiter E, Karatosun V, Günal I. Do orthopaedic journals provide high-quality evidence for clinical practice? Arch Orthop Trauma Surg. 2003;123(2-3):82-5.

47. Robert IE, Hyde CJ, Bridgman SA. Orthopaedic fractures: trends in randomised controlled trials. Injury. 2001;32(10):779-82.

48. Wright JG, Gebhardt MC. Multicenter clinical trials in orthopaedics: time for musculoskeletal specialty societies to take action. J Bone Joint Surg Am. 2005;87(1):214-7. 